\title{
Nano-laminated thin film metallic glass design for outstanding mechanical properties
}

\author{
P. Kontis ${ }^{\mathrm{a},{ }^{*},}$, M. Köhler ${ }^{\mathrm{a}}$, S. Evertz ${ }^{\mathrm{b}}$, Y.-T. Chen ${ }^{\mathrm{b}}$, V. Schnabel ${ }^{\mathrm{b}}$, R. Soler ${ }^{\mathrm{a}}$, J. Bednarick $^{\mathrm{c}}$,
} C. Kirchlechner ${ }^{\mathrm{a}}$, G. Dehm ${ }^{\mathrm{a}}$, D. Raabe ${ }^{\mathrm{a}}$, J. M. Schneider ${ }^{\mathrm{b}}$, B. Gault ${ }^{\mathrm{a}}$

\author{
${ }^{a}$ Max-Planck-Institut für Eisenforschung, Max-Planck-Str. 1, 40237 Düsseldorf, Germany. \\ ${ }^{\mathrm{b}}$ Materials Chemistry, RWTH Aachen University, Kopernikusstr. 10, D-52074 Aachen, \\ Germany \\ ${ }^{c}$ Deutsches Elektronen Synchrotron DESY, FS-PE group, Notkestrasse 85, D-22607 \\ Hamburg, Germany
}

*Corresponding author: p.kontis@mpie.de

\begin{abstract}
We report the enhancement of fracture toughness and strength of a cobalt-tantalum-based metallic glass thin film with increasing boron content. The improvement of the mechanical performance is attributed to the formation of a compositionally lamellar compared to uniform glass microstructure, which becomes thinner with increasing boron content as revealed by transmission electron microscopy. Compositional variations across the lamellar structure are revealed by atom probe tomography. Cobalt- and boron-rich regions alternate sequentially, whereas tantalum exhibits slight variations across the lamellae. Our results can be utilized in future design efforts for metallic glass thin films with outstanding mechanical performance.
\end{abstract}

Keywords: thin film metallic glasses, hybridization, fracture toughness, atom probe tomography

Metallic glasses exhibit a combination of high strength and toughness which make them an interesting material class for applications such as micro-gears, for instance in high precision endoscopes [1,2]. However, as these medical tools continuously decrease in size, micro-gears need to decrease in size accordingly, resulting in increased applied stresses during operation. In order to reduce the risk of brittle failure, tougher metallic glasses with high strength are required in that context [3].

For the design of tough metallic glasses, a universal relationship between Poisson's ratio and brittle-ductile transition has been suggested by Lewandowski et al. in 2005 [4]. However, as only the elastic behavior of metallic glasses is considered by the Poisson's ratio, there is an ongoing discussion in literature about the reliability of this criterion regarding ductility [5-8]. Based on theoretical and experimental data of $\mathrm{Co}-\mathrm{Cu}$ and Pd-based metallic glass systems, we have recently demonstrated that Poisson's ratio alone is not a universal predictor of the brittleductile transition. Instead we have proposed a design concept for damage-tolerant metallic glasses, where the low fraction of bonds stemming from hybridized states compared to the overall bonding serves as a fingerprint for damage tolerance [9]. While this assessment is based on a theoretical and experimental appraisal of homogeneous metallic glass systems which are without exception consistent with the notion put forward in Schnabel et al.[9], the here reported nano-lamellar Co-based metallic glass exhibiting a record fracture strength cannot be predicted based on any design notion available today. 
To evaluate the influence of metal-metalloid bonding on the stiffness and damage-tolerance of metallic glasses, we have systematically investigated the influence of Co-B bonds in combinatorial Co-Ta-B metallic glass thin films (MGTFs) by experimental and theoretical methods. In this study, Co-Ta-B MGTFs were magnetron sputtered on Si (100) wafers with a diameter of $50.8 \mathrm{~mm}$ for chemical and mechanical characterization as well as on polyimide substrates for synchrotron X-ray diffraction for topology analysis [10]. Data from 2D-XRD analysis of a representative film and with a substrate is shown in Supplementary Figure 1. A lab-scale ultrahigh vacuum chamber was utilized for the production of the MGTFs and it was operated at a base pressure in the range of $5 \cdot 10^{-6} \mathrm{~Pa}$. A $\mathrm{Co}_{88} \mathrm{Ta}_{12}$ and a $\mathrm{B}$ target were used for the deposition with a purity of $99.9 \%$ and $99.5 \%$, respectively. The magnetrons were tilted $45^{\circ}$ from the substrate normal and no substrate rotation was employed. For the CoTa compound and $\mathrm{B}$ elemental targets, a direct current and radio frequency power supply were used, respectively. The power densities applied were $0.4 \mathrm{~W} / \mathrm{cm}^{2}$ for the CoTa target and $8.4 \mathrm{~W} / \mathrm{cm}^{2}$ for the B target. A film thickness of $2.5 \mu \mathrm{m}$ was obtained.

Micro-cantilever bending experiments were carried out in situ in a scanning electron microscope (JEOL-JSM 2000) equipped with an ASMEC UNAT-2 indenter. 80 cantilevers were micro-machined using a focused ion-beam (FIB) microscope (FEI Helios Nanolab 600i dual-beam FIB) following the procedure described in Ref. [11]. A schematic illustration of the lamellae orientation with respect to nanoindentation and cantilever tests is shown in the Supplementary Figure 2. Prior to FIB milling, a free-standing film was obtained by selectively etching away the Si substrate, using a $30 \% \mathrm{KOH}$ solution at $80^{\circ} \mathrm{C}$ for $45 \mathrm{~min}[12,13]$. Five unnotched and five pre-notched cantilevers with size $18 \times 2.5 \times 2.5 \mu \mathrm{m}^{3}$ in size were fabricated and tested for eight different compositions to measure fracture strength and fracture toughness.

Bending tests were carried out in displacement-controlled mode using a constant displacement rate of $5 \mathrm{~nm} / \mathrm{s}$. A conical tip ( $1 \mu \mathrm{m}$ tip radius) was employed for the experiments. Fracture toughness, $\mathrm{K}_{\mathrm{IC}}$, was evaluated following linear elastic fracture mechanics (LEFM):

$$
K_{I C}=\frac{F_{\max } L}{B w^{\frac{3}{2}}} f\left(\frac{a}{w}\right)
$$

where $F_{\max }$ is the maximum load before fracture, $L$ is the beam length, $B$ the beam width, $w$ the beam thickness, and $a$ the notch depth of the pre-notch cantilevers. The function $f(a / w)$ is a shape factor and was determined by Matoy et al. [14] using FEM simulations. Cantilevers without pre-notch were used to calculate the fracture strength, $\sigma_{f}$, evaluated following classical bending beam theory:

$$
\sigma=6 \frac{F_{\max } l}{B w^{2}}
$$

For the structural and chemical characterization of the MGTFs transmission electron microscopy (TEM) and atom probe tomography (APT) were utilized. Samples for TEM investigation were fabricated with an FEI Helios Nanolab 600 followed by a post thinningprocess with illumination of $500 \mathrm{eV}$ ion beam. Micrographs were recorded in an FEI Titan G2 80-200 CREWLEY at $200 \mathrm{kV}$ with a high angle annular dark field (HAADF) detector with camera length of $110 \mathrm{~mm}$, and a device controlling system of DigiScan and Digital Micrograph. Site-specific 3D-APT samples were prepared using an FEI Helios Nanolab 600i dual-beam FIB. The APT specimens were prepared following the standard lift-out process [15]. The APT measurements were performed on a commercial CAMECA local electrode atom probe LEAP $3000 \mathrm{X} \mathrm{HR}$, in voltage mode at a base temperature of $60 \mathrm{~K}$, pulse repetition rate of $200 \mathrm{kHz}$, pulse fraction of $15 \%$. 
Topological analysis was performed by synchrotron X-ray diffraction at beamline P02.1 at the electron storage ring PETRA III (DESY, Hamburg, Germany). The X-ray diffraction was carried out in transmission along the CoTa-B compositional gradient of the MGTFs deposited on polyimide foil [16]. A monochromatic photon beam with a spot size of $0.7 \mathrm{~mm} \times 0.7 \mathrm{~mm}$ and a wavelength of $0.02070 \mathrm{~nm}$ was used. The diffracted 2D patterns were recorded with a fast image plate detector Perkin Elmer 1621, positioned at a distance of $238 \mathrm{~mm}$ from the thin film. The sample to detector distance, orthogonality of the detector and beam centre was calibrated using a $\mathrm{CeO}_{2}$ powder standard (National Institute of Standards and Technology 674b). Individual diffraction patterns were acquired for 30s. The diffraction patterns were integrated into q-space $(q=4 \pi \sin \theta / \lambda)$ up to $18 \AA^{-1}$ using the FIT2D software package [17]. The pair distribution functions (PDF) were obtained from the integrated diffraction intensity after correction for background contributions, sample absorption, inelastic scattering and normalization to the atomic X-ray form factor employing the PDFgetX2 software [18].

Finally, ab initio molecular dynamic simulations with the density functional theory [19] based openMX code [20,21] were carried out. Electronic potentials with the generalised gradient approximation were employed [22]. Basis functions were linear combinations of localised pseudoatomic orbitals [23]. The following basis functions were applied: Co5.5-s2p1d1, Ta7.0s2p1d1f1, B4.5-s2p2. The first symbol designates the chemical element followed by the cutoff radius. The last set of symbols defines the primitive orbitals. An N-point grid of $85 \times 85 \times 85$ and a cutoff energy of $150 \mathrm{Ry}$ has been used. For volume relaxation at $0 \mathrm{~K}$ the Vienna Abinitio Simulation Package was utilised $[24,25]$. Thereby, the ultrasoft pseudopotentials were employed and the Brillouin zone was integrated on a 3x3x3 Monkhorst-Pack k-point grid [26]. To model the short range ordered structure of the metallic glass, the structural model introduced by Hostert et al. [27] was applied with a supercell containing 115 atoms and 13 vacancies. In order to obtain an amorphous structure, the supercell was heated to $4000 \mathrm{~K}$ for $4000 \mathrm{fs}$ and quenched to $0 \mathrm{~K}$ with infinite cooling rate until the volume change between two subsequent cycles was below $2 \%$.

Fig. 1 shows the micro-mechanical behaviour of the MGTF as measured along the boron gradient. Micro-cantilever bending tests revealed an increase in fracture strength and fracture toughness as the boron content increases, Figure 1a. For the material with the highest boron content probed, the fracture strength is in the range of $11 \pm 0.4 \mathrm{GPa}$, i.e. twice as high as that observed for the ultra-high strength $\mathrm{Co}_{43} \mathrm{Fe}_{20} \mathrm{Ta}_{5.5} \mathrm{~B}_{31.5}$ bulk metallic glass with a fracture strength of $5.2 \mathrm{GPa}$ reported by Inoue et al. [3]. A fracture toughness of $6 \mathrm{MPa} \sqrt{\mathrm{m}}$ is achieved for the material with highest boron composition and $2 \mathrm{MPa} \sqrt{\mathrm{m}}$ for the lowest $\mathrm{B}$ content. Fig. $1 \mathrm{~b}$ shows the hardness and reduced Young's modulus as a function of boron content as revealed by nanoindentation measurements. Both the reduced Young's modulus and hardness increase as boron content increases. For the highest investigated boron content, the reduced Young's modulus reaches a value of $344 \pm 1.5 \mathrm{GPa}$, which is slightly higher than the record Young's modulus of $309 \mathrm{GPa}$ reported by Ohtsuki et al. [28] for a $\mathrm{W}_{46} \mathrm{Re}_{37} \mathrm{~B}_{17}$ metallic glass. Representative fracture surfaces and load-deflection curves are given in the Supplementary Figure 3 and 4, respectively.

To understand the superior stiffness, a short-range order analysis for $\mathrm{Co}_{61.0} \operatorname{Ta}_{11.0} \mathrm{~B}_{28.0}$, $\mathrm{Co}_{55.4} \mathrm{Ta}_{9.7} \mathrm{~B}_{35.0}$ and $\mathrm{Co}_{43.7} \mathrm{Ta}_{6.8} \mathrm{~B}_{49.5}$ was performed. Fig. 2 shows the real space pair distribution functions (PDF). For the $\mathrm{Co}_{61.0} \mathrm{Ta}_{11.0} \mathrm{~B}_{28.0}$ the maxima of the first order metal to boron bond population at a distance of $2.02 \AA$ is marked by a vertical dashed line. The first order metal to metal bond population is within the range of 2.2 to $3.4 \AA$, with its maxima marked by a second vertical line. Furthermore, the PDF analysis in Fig. 2 reveals a continuous 
shift of the metal-metal and metal-boron bond population maxima with increasing boron content. The shift corresponds to a relative increase of $2.0 \%$ and $0.8 \%$ for the metal to boron and metal to metal bond distance, respectively. This increase in bond distance can be attributed to a similar weakening of the boron induced metallic bond as reported for Co-B based metallic glasses [16,29]. The PDF also shows a boron-induced increase in the first order metal-boron bond population corresponding to an increase in density of strong metal-to-boron bonds [27,30]. Based on the reports by Schnabel et al. [16,31], an increase in boron content results in a concomitant increase of bond density as well as bond energy density [31]. Hence, the origin of the ultrahigh stiffness reported here in Fig. 1is best understood in terms of the bond energy density [31].

To rationalize the observed micro-mechanical performance in terms of fracture strength and toughness, STEM and APT were performed specifically at the compositions $\mathrm{Co}_{55.4} \mathrm{Ta}_{9.7} \mathrm{~B}_{35.0}$ and $\mathrm{Co}_{43.7} \mathrm{Ta}_{6.8} \mathrm{~B}_{49.5}$ that showed a large difference in mechanical properties. HAADF-STEM analysis was initially conducted, where contrast arises due to changes in the average atomic weight. Fig. $3 \mathrm{a}$ and $3 \mathrm{~b}$ show a lamellar structure inclined by approximately $20^{\circ}$ with respect to the film growth axis and with compositional variations for both compositions, $\mathrm{Co}_{55.4} \mathrm{Ta}_{9.7} \mathrm{~B}_{35.0}$ and $\mathrm{Co}_{43.7} \mathrm{Ta}_{6.8} \mathrm{~B}_{49.5}$. The lamellar structure becomes thinner and more refined for the MGTF with higher bulk boron content. These compositional variations are confirmed through the application of APT that shows a succession of boron enriched and boron depleted regions as shown in Fig. 3c-3f, for each individual bulk composition. In Fig. 3e and 3f, slight variations of tantalum across the lamellar structure was observed. STEM and APT analysis of regions with a boron content below 28 at.\% shows a completely uniform microstructure with uniform distribution of cobalt, tantalum and boron.

These record fracture strength of the here reported nano-lamellar Co-based metallic glass thin film cannot be understood by any design notion entertained today. With a Poisson's ratio of 0.33 and 0.30 for $\mathrm{Co}_{55} \mathrm{Ta}_{10} \mathrm{~B}_{35}$ and $\mathrm{Co}_{47} \mathrm{Ta}_{6} \mathrm{~B}_{47}$, respectively, $\mathrm{Co}_{55} \mathrm{Ta}_{10} \mathrm{~B}_{35}$ is close to the toughbrittle transition and $\mathrm{Co}_{47} \mathrm{Ta}_{6} \mathrm{~B}_{47}$ clearly in the brittle regime, assuming the tough-brittle transition proposed by Lewandowski et al. [32]. Since the fingerprint for toughness can be found in the electronic structure [9], the electronic density of states (DOS) of $\mathrm{Co}_{55} \mathrm{Ta}_{10} \mathrm{~B}_{35}$ is shown in Fig. 4. The upper panel shows the total DOS, while the lower panels show the partial DOS of the constituents Co, Ta and B. The Fermi level is shifted to $0 \mathrm{eV}$. The $\mathrm{d}$ bands of Co and Ta are strongly overlapping between -6 and $0 \mathrm{eV}$. Comparing the $\mathrm{d}$ bands of Co and Ta with the shape of the $\mathrm{B} p$ band between -6.5 and $-2 \mathrm{eV}$, an overlap between the $\mathrm{p}$ band of $\mathrm{B}$ and the $\mathrm{d}$ band of $\mathrm{Co}$ and Ta is visible. Therefore, a strong contribution of hybridised bonds to the overall bonding in $\mathrm{Co}_{55} \mathrm{Ta}_{10} \mathrm{~B}_{35}$ is expected. Thus, stiff behaviour of the material (with limited toughness) due to the prohibition of shear relaxation and of the formation of shear transformation zones is predicted. It may be speculated that the observed high fracture strength may be enabled by the nano-laminated (lamellar) architecture, whose origin is not understood yet and subject of ongoing work.

As the formation of lamellar structures within metallic glasses seems to result in excellent fracture strengths, it can be used as a design route for excellent mechanical performance. This design route is controlled by the process parameters of the PVD process. Phase separation occurring in the liquid during cooling of bulk metallic glasses is normally achieved with a large positive heat of mixing [33]. However, in the current example calculations of the enthalpies of mixing by the Calphad approach show that all enthalpies of mixing are negative and also the differences of the interaction parameters are lower than reported for phase separation for metallic glasses in literature [34]. The cohesive energy for $\operatorname{Co}_{55.4} \mathrm{Ta}_{9.7} \mathrm{~B}_{35.0}$ was 
estimated by ab initio calculations. For a homogeneous supercell, a cohesive energy of $7.444 \mathrm{eV} /$ atom was found. Based on the input from the APT measurements, a computational supercell was created containing a boron-rich $\mathrm{Co}_{50} \mathrm{Ta}_{10} \mathrm{~B}_{40}$ and a cobalt-rich $\mathrm{Co}_{60} \mathrm{Ta}_{10} \mathrm{~B}_{30}$ half, leading to a cohesive energy of $-7.447 \mathrm{eV} / \mathrm{atom}$. The energy difference of $3 \mathrm{meV} / \mathrm{atom}$ or $0.04 \%$ is not significant, indicating that both phases are thermodynamically equally (meta)stable. As the formation of the lamellar structure cannot be interpreted by thermodynamics, it is believed that its formation is kinetically driven. This is in agreement with observations made on completely DC sputtered samples of same composition where a homogeneous structure was observed, compared to the lamellar structure found in the MGTF, where boron was RF sputtered.

In summary, our findings provide a new design route for a cobalt-boron-based metallic glass thin films with enhanced mechanical performance as a function of boron content. A compositional lamellar structure revealed by TEM shows that the lamella thickness decreases as boron content increases. A substantial compositional difference was observed within the lamellar structure, where boron- and cobalt-rich structures were revealed by atom probe tomography. As the thickness of the lamellar structure decreases, fracture toughness and fracture strength are enhanced, suggesting the origin of the enhanced mechanical properties in the nanolaminated structure. The origin of the lamella formation is subject of ongoing research.

\section{Acknowledgements}

The authors acknowledge support by the German National Science Foundation (DFG) within the SPP-1594 (DE796/9-2, RA 659/28-2 and SCHN 735/35-2). Simulations were performed with computing resources granted by JARA-HPC from RWTH Aachen University under project JARA0131. Uwe Tezins and Andreas Sturm are also acknowledged for their support on APT experiments. Parts of this research were carried out at beamline P02.1 of the light source PETRA III at DESY, a member of the Helmholtz Association (HGF).

\section{References}

[1] A. Inoue, B. Shen, A. Takeuchi, Mater. Trans. 47 (2006) 1275-1285.

[2] M. Ishida, H. Takeda, D. Watanabe, K. Amiya, N. Nishiyama, K. Kita, Y. Saotome, A. Inoue, Mater. Trans. 45 (2004) 1239-1244.

[3] A. Inoue, B.L. Shen, H. Koshiba, H. Kato, A.R. Yavari, Acta Mater. 52 (2004) 16311637.

[4] J.J. Lewandowski, W.H. Wang, A.L. Greer, Philos. Mag. Lett. 85 (2005) 77-87.

[5] G. Kumar, P. Neibecker, Y.H. Liu, J. Schroers, Nat. Commun. 4 (2013) 1536.

[6] Y.Y. Zhao, A. Inoue, C. Chang, J. Liu, B. Shen, X. Wang, R.W. Li, Sci Rep 4 (2014) 5733.

[7] N. Zheng, R.T. Qu, S. Pauly, M. Calin, T. Gemming, Z.F. Zhang, J. Eckert, Appl. Phys. Lett. 100 (2012) 141901.

[8] R. Raghavan, P. Murali, U. Ramamurty, Acta Mater. 57 (2009) 3332-3340.

[9] V. Schnabel, B.N. Jaya, M. Köhler, D. Music, C. Kirchlechner, G. Dehm, D. Raabe, J.M. Schneider, Sci. Rep. 6 (2016) 36556.

[10] J. Megusar, J. Nucl. Mater. 245 (1997) 185-190.

[11] B.N. Jaya, C. Kirchlechner, G. Dehm, J. Mater. Res. 30 (2015) 686-698.

[12] K. Biswas, S. Kal, Microelectronics J. 37 (2006) 519-525. 
[13] A. Riedl, R. Daniel, J. Todt, M. Stefenelli, D. Holec, B. Sartory, C. Krywka, M. Müller, C. Mitterer, J. Keckes, Surf. Coatings Technol. 257 (2014) 108-113.

[14] K. Matoy, H. Schönherr, T. Detzel, T. Schöberl, R. Pippan, C. Motz, G. Dehm, Thin Solid Films 518 (2009) 247-256.

[15] K. Thompson, D. Lawrence, D.J. Larson, J.D. Olson, T.F. Kelly, B. Gorman, Ultramicroscopy 107 (2007) 131-139.

[16] V. Schnabel, M. Köhler, S. Evertz, J. Gamcova, J. Bednarcik, D. Music, D. Raabe, J.M. Schneider, Acta Mater. 107 (2016) 213-219.

[17] A.P. Hammersley, S.O. Svensson, M. Hanfland, A.N. Fitch, D. Hausermann, High Press. Res. 14 (1996) 235-248.

[18] X. Qiu, J.W. Thompson, S.J.L. Billinge, J. Appl. Crystallogr. 37 (2004) 678-678.

[19] P. Hohenberg, W. Kohn, Phys. Rev. 136 (1964) B864-B871.

[20] T. Ozaki, H. Kino, Phys. Rev. B 69 (2004) 195113.

[21] T. Ozaki, H. Kino, Phys. Rev. B 72 (2005).

[22] J.P. Perdew, K. Burke, M. Ernzerhof, Phys. Rev. Lett. 77 (1996) 3865-3868.

[23] T. Ozaki, Phys. Rev. B 67 (2003) 155108.

[24] G. Kresse, J. Furthmüller, Phys. Rev. B 54 (1996) 11169-11186.

[25] G. Kresse, D. Joubert, Phys. Rev. B 59 (1999) 1758-1775.

[26] H.J. Monkhorst, J.D. Pack, 13 (1976).

[27] C. Hostert, D. Music, J. Bednarcik, J. Keckes, V. Kapaklis, B. Hjörvarsson, J.M. Schneider, J. Phys. Condens. Matter 23 (2011) 475401.

[28] M. Ohtsuki, R. Tamura, S. Takeuchi, S. Yoda, T. Ohmura, Appl. Phys. Lett. 84 (2004) 4911-4913.

[29] M.L. Cohen, Phys. Rev. B 32 (1985) 7988-7991.

[30] J. Wang, R. Li, N. Hua, T. Zhang, J. Mater. Res. 26 (2011) 2072-2079.

[31] J. Phys. Condens. Matter 29 (n.d.).

[32] J.J. Lewandowski, W.H. Wang, A.L. Greer, Philos. Mag. Lett. 85 (2005) 77-87.

[33] A.L. Greer, Y.Q. Cheng, E. Ma, Mater. Sci. Eng. R Reports 74 (2013) 71-132.

[34] T. Abe, M. Shimono, K. Hashimoto, K. Hono, H. Onodera, Scr. Mater. 55 (2006) 421424. 


\section{Figures}

a)

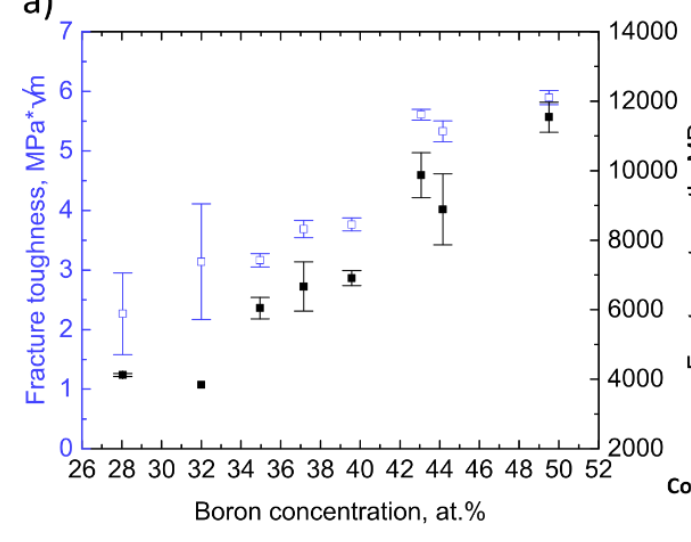

b)

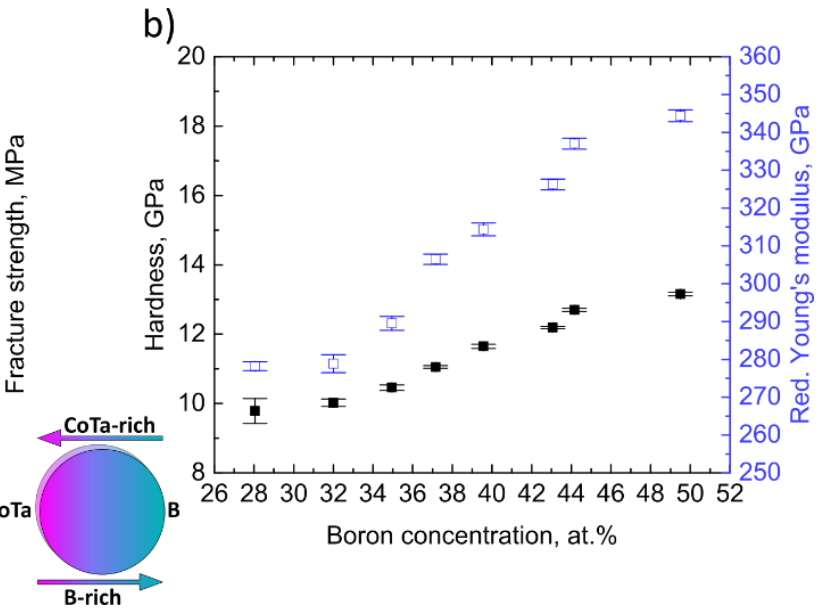

Figure 1: Micro-mechanical testing of the combinatorial CoTaB metallic glass thin film. a) Fracture toughness and fracture strength graphs as a function of boron content as revealed by micro-cantilever bending tests. b) Young's modulus and hardness as a function of boron content measured by nanoindentation. The error bars correspond to the standard deviation.

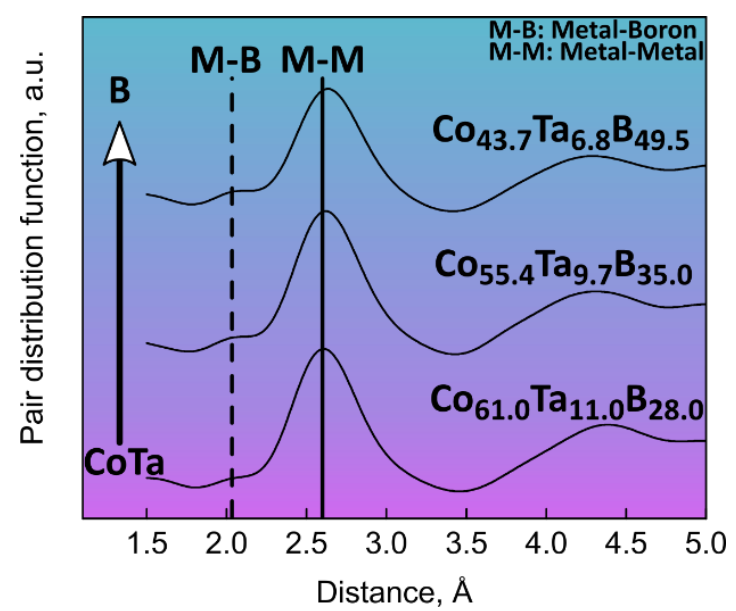

Figure 2: Pair distribution functions with increasing B-content from bottom to top as obtained by high energy X-ray diffraction are presented in a range between 1.5 and $5.0 \AA$. 

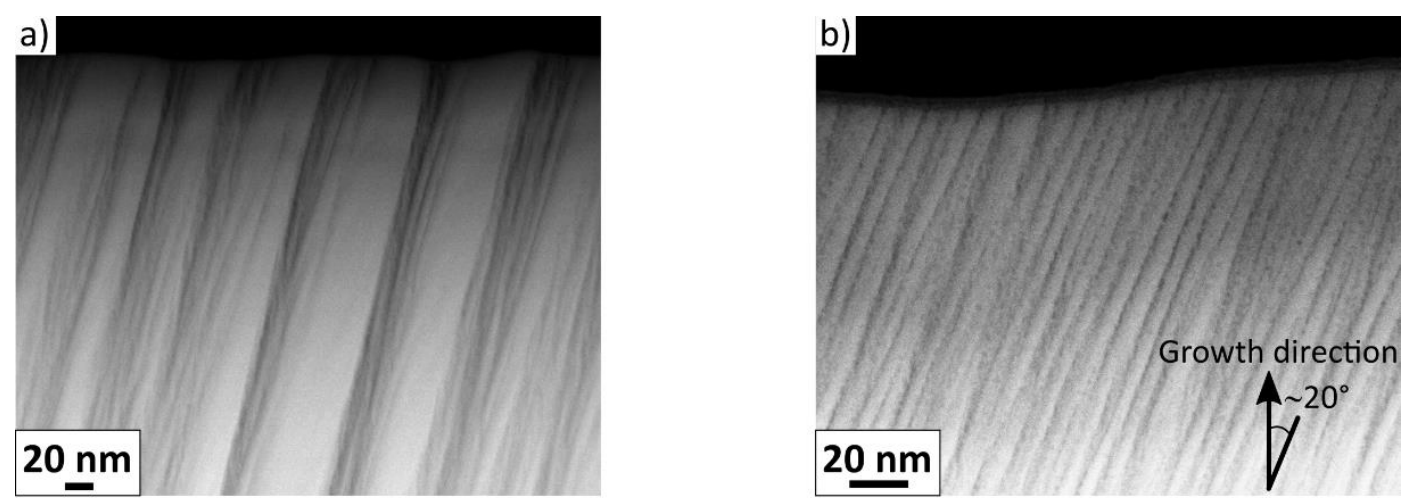

\section{$\mathrm{Co}_{55.4} \mathrm{Ta}_{9.7} \mathrm{~B}_{35.0}$}

c)
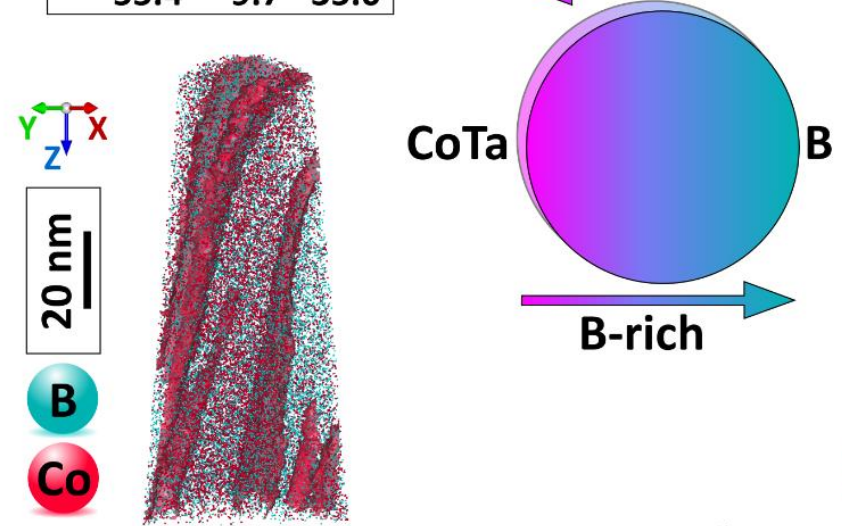

$\mathrm{Co}_{43.7} \mathrm{Ta}_{6.8} \mathrm{~B}_{49.5}$
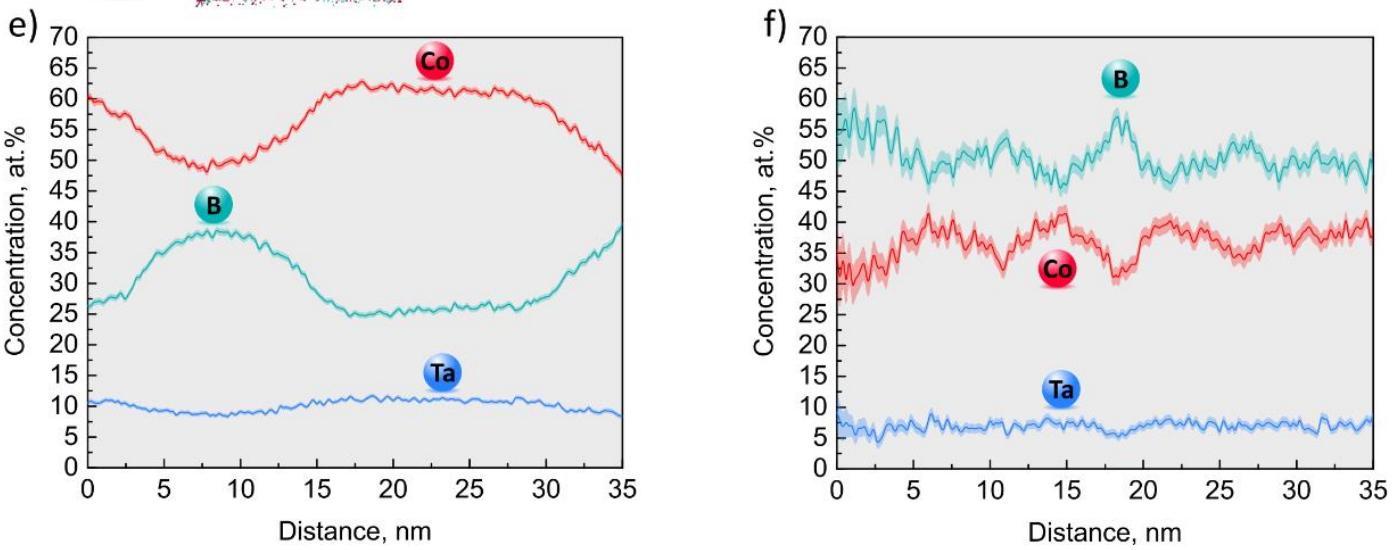

Figure 3: a,b) HAADF-STEM micrographs revealing the lamellar structure for $\mathrm{Co}_{55.4} \mathrm{Ta}_{9.7} \mathrm{~B}_{35.0}$ and $\left.\mathrm{Co}_{43.7} \mathrm{Ta}_{6.8} \mathrm{~B}_{49.5} \mathrm{c}, \mathrm{d}\right)$ Corresponding APT reconstructions from $\mathrm{Co}_{55.4} \mathrm{Ta}_{9.7} \mathrm{~B}_{35.0}$ and $\mathrm{Co}_{43.7} \mathrm{Ta}_{6.8} \mathrm{~B}_{49.5}$ compositions. e,f) Compositional profiles perpendicular to lamellar structures revealing the boron and cobalt variations. 


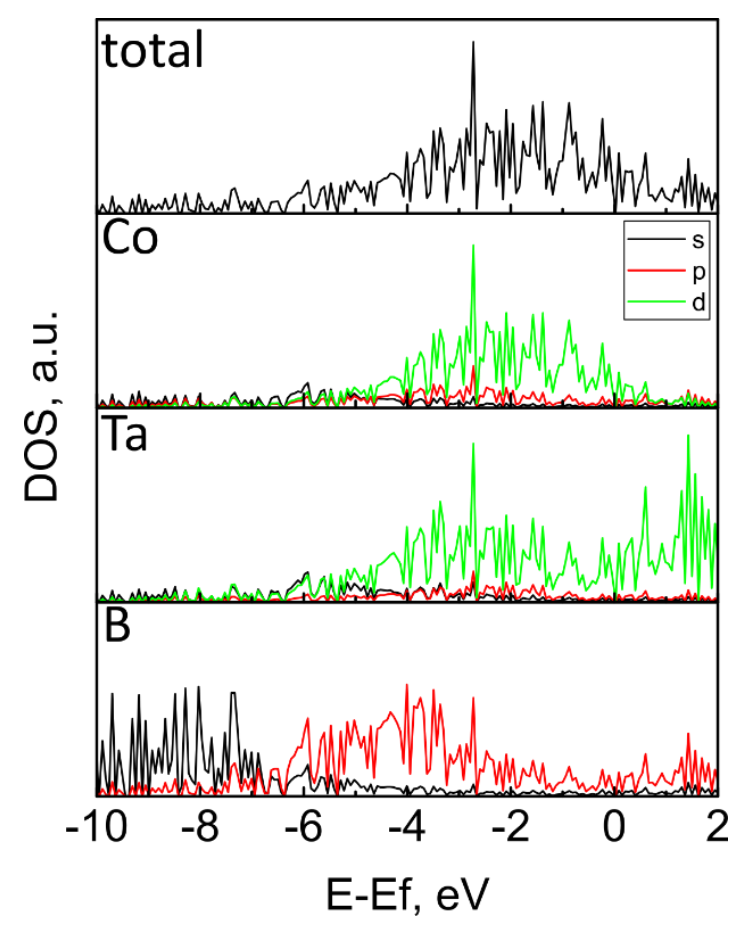

Fig. 4: Electronic density of states for CoTaB35. Total DOS in the top panel, below the partial DOS for Co, Ta and B. The Fermi level is shifted to $0 \mathrm{eV}$.

\section{Supplementary Figures}

a) b)

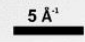

Sup. Fig. 1: a) 2D XRD patterns of the metallic glass thin film with the highest boron content and b) with the substrate. For better visibility, q-space is only displayed up to about $15 \AA^{-1}$.

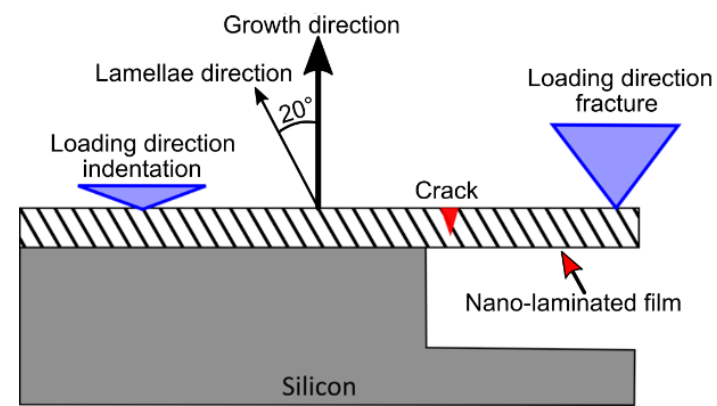

Sup. Fig. 2: Schematic illustration of the lamellae orientation with respect to nanoindentation and cantilever tests. 


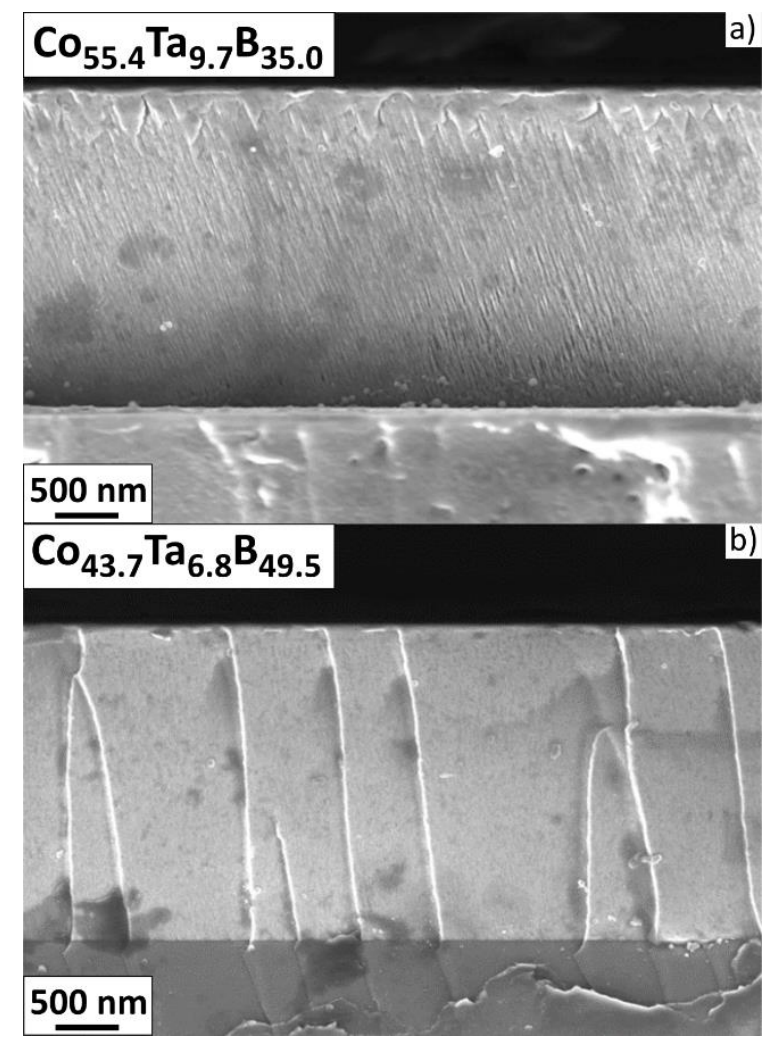

Sup. Fig. 3: Fracture surface from cantilever testing for $\mathrm{Co}_{55.4} \mathrm{Ta}_{9.7} \mathrm{~B}_{35.0}$ and $\mathrm{Co}_{43.7} \mathrm{Ta}_{6.8} \mathrm{~B}_{49.5}$.

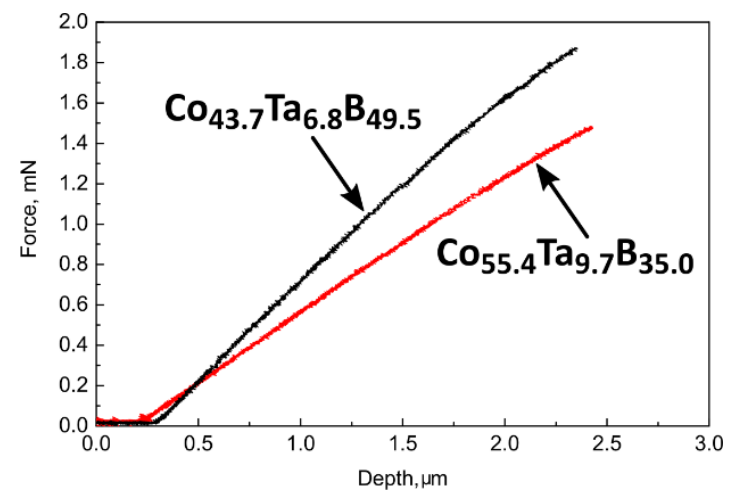

Sup. Fig. 4: Load-deflection curves for $\mathrm{Co}_{55.4} \mathrm{Ta}_{9.7} \mathrm{~B}_{35.0}$ and $\mathrm{Co}_{43.7} \mathrm{Ta}_{6.8} \mathrm{~B}_{49.5}$ 